\title{
Does Time of Day Effect Insect Oviposition
}

Robin Marriott ${ }^{1^{*}}$

University of Leicester, Nottingham, Nottinghamshire, United Kingdom

*Corresponding author: Robin Marriott, University of Leicester, Nottingham, Nottinghamshire, United Kingdom, Tel: 07983513204; E-mail: robin.w.marriott@gmail.com Received date: December 22, 2017; Accepted date: January 09, 2018; Published date: January 16, 2018

Copyright: (c) 2018 Marriott R, This is an open-access article distributed under the terms of the Creative Commons Attribution License, which permits unrestricted use, distribution, and reproduction in any medium, provided the original author and source are credited.

Keywords: Nocturnal; Oviposition; Blowflies; Carcasses

\section{Short Communication}

The nocturnal activity of blowflies and their ability to oviposit during the night and in the dark is an ongoing debate but it is however generally thought of that blowflies are not active at night and do not lay eggs at night [1]. Despite this, some argue against this suggesting that blowflies are indeed active at night and oviposit at night and in the dark [2]. With this in mind, this paper presents an argument to support and provide evidence of the active nature of blowflies at night and their ovipositing habits. Areas such as the effect of artificial light on blowfly oviposition and nocturnal blowfly activity are discussed from different perspectives in order to present a significant argument.

Firstly it is important to examine the general belief of the limited ovipositing activity of blowflies at night of which there is much research on the topic. For example, a study by Baldridge, et al. [3] involved recording ovipositing nocturnal activities of flies during the hours between 2100-0600. Carrion used in the experiment included, freshly killed rats, fresh and aged beef and freshly thawed pigs. Results of the study showed that the flies that were recorded did not oviposit during the night. Reinforcing this research, Stamper et al, [4] provided a study where by blowflies' activity at night, in both rural and urban locations which were li and unlit, and of which were exposed to euthanized rats, were monitored. Similar results were provided as nocturnal oviposition of the blowflies was not apparent.

Although such research provides a clear result regarding the activity and oviposition of blow flies at night, the use of carcasses in the studies need to be addressed. For example, the use of rat carcasses may have an impact on the experiment as due to the lack of resources available on such as small carcass, some species of fly will not colonize it [5]. Furthermore, in contrast to the study by Stamper, et al. [4], research conducted by Greenberg [6] showed that Calliphora vicina (RobineauDesvoidy), $P$. sericata, and Phormia regina (Meigen) did indeed oviposit during night time where artificial lighting may simulate daylight hours. However, it is argued that the placement bait used in the study to attract the blowflies was placed in position whereby resting blowflies that were nearby could easily have climbed to the bait and therefore questions the actual attraction and activity of the flies at night [7]. As a result of this, research by Singh and Bharti [7] build upon the study by Greenberg [6] and provide further evidence to highlight the activity of blowflies during nocturnal hours. The study involved placing mutton at a height above the ground at night and away from any sources of light or illumination. Results provided similar evidence as to that of the study conducted by Greenberg [6] as calliphorid flies were recorded to have laid eggs at night as well as in the day time. Results did indicate that during daytime hours, more eggs were laid rather that at night time. However, evidence of nocturnal oviposition of blowflies is still evident.
Some laboratory studies however, provide evidence against this and provide evidence that suggest blowflies have a limited ability to be active in the dark. For example Wooldridge, et al. [8] provided a study to which claims that light intensity correlates with the activities of blowflies and claim that due to this, it is highly unlikely that blowflies will oviposit in the dark. However, in contrast to this, a study conducted by Faucherre, et al. [9] consisted of placing fresh meat and pork liver in a 10 meter deep cave where it was dark. Results of this showed that after checking the meat and liver, eggs had been laid of which later were reared to into adults belonging to $C$. vicina. As a result of this study, it can be argued that blowflies are active during darkness and the probability of oviposition does not therefore depend on light intensity. Furthermore, some laboratory studies conducted also support the notion of the activity of blowflies at night and in the dark. For example, laboratory studies produced findings to show that, in the southern hemisphere, L. sericata, L. cuprina, Chrysomya chloropyga and Chrysomya putoria were recorded to have laid eggs in a dark environment. Furthermore, evidence showed that the rate of eggs that were laid in nocturnal conditions increased as the ambient temperature increased of which suggests that temperature has an impact on oviposition rather than light intensity $[10,11]$ reinforces this claiming that, aroused gravid females, on a night where by the temperature is high, may indeed oviposit. However Amendt, et al. [11] do also argue that this is an unlikely event.

Although it is claimed that blowfly oviposition is unlikely during the night and in the dark, it is clear to see that some studies show that oviposition is likely. As a result of this PMI estimations can be dramatically alter. For example Greenberg [6] claims that nocturnal oviposition can alter an estimation based on limited nocturnal activity of blowflies by as much as 12 hours. A study to highlight the importance of nocturnal activity in regard to PMI involved scuttle flies of which are known to be of forensic importance when indoors as they are known to show diurnal and nocturnal behaviour. Scuttle fly traps were placed within a laboratory setting and consisted of cow's liver. The traps were then divided between light exposed conditions and dark conditions to provide data to compare. Results showed that all the specimens that were collected showed activity and oviposition during both light expose and complete darkness and therefore suggest that nocturnal oviposition should be accounted for when estimating PMI [12].

Although the subject of nocturnal activity and oviposition of blowflies is contradictory within research, it is clear to see that there are many different studies and laboratory experiments that do provide evidence to suggest that blowflies are active at night time and also in darkness. Furthermore, it can also be argued that light intensity is not the stimulus required for blow flies to oviposit and that research regarding temperature and nocturnal activities of blowflies should be more closely examined. It is also clear to see that the consequences of not taking the notion of nocturnal blowfly activity can be dramatic and 
make a significant difference when regarding circumstances such as PMI. Therefore, it can be argued that although the studies used in this paper provide contrasting results, the possible activities and oviposition of blowflies in the dark and at night should always be examined and taken into account when concerning PMI.

\section{References}

1. Greenberg B, Kunich J (2002) Entomology and the law, flies as forensic indicators. Cambridge: Cambridge University Press.

2. Hui Y (2003) Food plant sanitation. New York: Marcel Dekker, Inc.

3. Baldridge RS, Wallace SG, Kirkpatrick R (2006) Investigation of nocturnal oviposition by necrophilous flies in central texas. J Forensic Sci 51: $125-126$

4. Stamper T, Davis P, DeBry RW (2009) The nocturnal ovipositing behavior of carrion flies in cincinnati, Ohio. J Forensic Sci 54: 1450-1452.

5. Benbow, E, Tomberlin JK, Tarone AM (2016) Carrion ecology, evolution and their applications. London: CRC Press.

6. Greenberg B (1990) Nocturnal oviposition behavior of blow flies (Diptera: Calliphoridae). J Med Entomol 27: 807-810.
7. Singh D, Bharti M (2001) Further observations on the nocturnal oviposition behaviour of blow flies (Diptera: Calliphoridae). Forensic Sci Int 120: 124-126.

8. Wooldridge J, Scrase L, Wall R (2007) Flight activity of the blowflies, Calliphora vomitoria and Lucilia sericata, in the dark. Forensic Sci Int 172: 94-97.

9. Faucherre, J (1999) Behavior of Calliphora vicina (Diptera, Calliphoridae) under extreme conditions. J Insect Behav 12: 687-690.

10. Williams KA, Wallman JF, Lessard BD, Kavazos CRJ , Mazungula DN, et al. (2017) Nocturnal oviposition behavior of blowflies (Diptera: Calliphoridae) in the southern hemisphere (South Africa and Australia) and its forensic implications. Forensic Sci Med Pathol 13: 123-134.

11. Amendt, J, Zehner R, Reckel F (2008) The nocturnal oviposition behaviour of blowflies (Diptera: Calliphoridae) in Central Europe and its forensic implications. Forensic Sci Int 175 :61-64.

12. Zulaikha A, Zuha M (2016) Nocturnal oviposition of the forensic scuttle fly, Megaselia scalaris (Loew) (Diptera: Phoridae) indoors. Egypt J Forensic Sci 6: 489-491. 